\title{
Reconocimiento de Caras y Discapacidad Intelectual
}

\section{Face Recognition and Learning Disability}

\author{
Antonio L. Manzanero \\ Universidad Complutense de Madrid, España \\ Alberto Alemany \\ Fundación Carmen Pardo Valcarce, España
}

\author{
María Recio \\ Fundación Carmen Pardo Valcarce, España \\ Almudena Martorell \\ Fundación Carmen Pardo Valcarce, España
}

\begin{abstract}
Resumen. El objetivo del presente trabajo consistió en analizar la capacidad de personas con discapacidad intelectual ligera y moderada para identificar a personas desconocidas vistas durante un periodo de tiempo breve y evaluar los factores que pudieran facilitar la tarea minimizando los errores. Para ello, se presentó a dos grupos de sujetos, con discapacidad y control, una fotografía de una persona que tras una tarea distractora deberían identificar en dos ruedas de seis fotografías (una de objetivo ausente y otra de objetivo presente). Cada sujeto realizó cuatro ensayos, dos con fotografías de hombres y otras dos con fotografías de mujeres. Los resultados mostraron que las personas con discapacidad intelectual en comparación con los sujetos control cometían menos aciertos y más falsas alarmas y discriminaban peor las caras (d'), aunque con pocas diferencias en el criterio de respuesta (ß). La relación entre rendimiento y CI resultó significativa para algunos de los tipos de respuesta.

Palabras clave: discapacidad intelectual, identificación de personas, memoria, psicología del testimonio.
\end{abstract}

\begin{abstract}
The aim of this study was to analyze the unfamiliar face recognition ability of people with mild and moderate intellectual disabilities and assess factors that might facilitate the task while minimizing errors. For this purpose, two groups had to recognize in two lineups with six photographs each (one present-target and one absent-target). Each subject performed four trials, two with pictures of men and two with women. Analysis of the results suggests that people with intellectual disabilities compared with control group made fewer hits and more false alarms and discriminate faces worst (d'), but with few differences in the response bias ( $($ ). The relationship between unfamiliar face recognition performance and IQ was significant in some types of responses.

Keywords: eyewitness testimony, face identification, intellectual disability, memory.
\end{abstract}

\section{Introducción}

No es infrecuente que personas con discapacidad intelectual (DI) sean víctimas de delitos (Goldman, 1994). Uno de los principales problemas de este tipo de casos reside en la identificación de los autores de

La correspondencia sobre este artículo debe enviarse al primer autor al e-mail: antonio.manzanero@psi.ucm.es los delitos cuando se trata de personas desconocidas para las víctimas. Ericson e Isaacs (2003) comparando adultos con DI y sin DI encontraron que ambos acertaban por igual en sus identificaciones, pero los primeros cometían más falsas alarmas y tendían más a adivinar que los segundos.

En cualquier caso, hay pocos estudios acerca de la capacidad que personas con distintos tipos de discapacidad cognitiva tienen a la hora de llevar a cabo la 
tarea de identificación en entornos judiciales y de los factores que pueden influir en su exactitud. La escasez de estudios, junto con los mitos asociados a las personas con DI, pueden llevar a no tener en cuenta las identificaciones realizadas por testigos o víctimas con DI. En el polo contrario, está la tendencia a equiparar a una víctima con DI a una víctima sin DI, sin tener en cuenta que con la primera se deben ajustar los procedimientos para obtener las máximas garantías y facilitar la tarea.

Una revisión de los estudios sobre discapacidad intelectual y procesos cognitivos muestra que el principal problema metodológico reside en la asignación de los sujetos a un grupo específico de características más o menos homogéneas que nos permitiera en un futuro generalizar los resultados y predecir comportamientos. La dificultad reside en que la inmensa mayoría de las personas consideradas como con discapacidad intelectual se encuadran en lo que podríamos denominar "inespecíficas". Así, aunque sólo una minoría de personas con DI podrían ser adscritas a una categoría específica determinada por el síndrome previamente diagnosticado (Down, Trastornos de Especto Autista, Rett, Prader-Willi, Williams-Beuren, etc.), la mayoría de los estudios se han centrado en analizar déficit cognitivos asociados a síndromes específicos. Por ejemplo, Annaz, Karmiloff-Smith, Johnson y Thomas (2004, 2009) establecen que existen diferencias en el rendimiento en tareas de identificación de caras entre Autismo, Síndrome de Down y Síndrome de Williams; y Lawrence, Kuntsi, Coleman, Campbell y Slarse (2003) afirman que hay un mayor déficit en el Síndrome de Turner.

Cuando hablamos de personas con discapacidad intelectual nos referimos a aquellas personas con "un funcionamiento intelectual general significativamente inferior a la media (CI menor de 70) asociado a un déficit del funcionamiento adaptativo actual en al menos dos ámbitos (comunidad, autocuidado, vida familiar, etc...) (Asociación Americana de Psiquiatría, 2009). Dado que los procesos cognitivos relevantes en la capacidad para realizar una identificación de una persona desconocida son fundamentalmente los de atención, percepción, memoria y lenguaje, y otros subprocesos o metaprocesos relacionados (Manzanero, 2006), podemos atrevernos a hipotetizar que la capacidad para identificar a una persona en aquellos contextos especialmente difíciles (persona desconocida, un tiempo de interacción escaso o una vista muy breve de la persona a identificar) puede estar más resentida en las personas con discapacidad intelectual que en las personas sin discapacidad intelectual.

En la medida en que las personas con DI tuvieran algún déficit en la capacidad de recuerdo, de distinguir realidad y fantasía, de atención, de percepción visual y auditiva, de expresión, de pensamiento y toma de decisiones, de localización espacio-temporal, etc., su capacidad para aportar un testimonio válido se resentiría. No obstante, dado que estos déficit podrían ir asociados tanto al tipo de trastorno como al nivel de afectación o discapacidad, no es posible generalizar, y por lo tanto sería oportuno alejarnos de los estereotipos y evaluar estos procesos para cada sujeto. Así, por ejemplo, conocemos que en general las personas con un trastorno de espectro autista (TEA) tendrán problemas a la hora de identificar a una persona (Behrmann, Avidan, Leonard, Kimchi, Luna, Humphreys y Minshew, 2006; Davies, Bishop, Manstead y Tantam, 1994; Gepner, De Gelder y Schonen, 1996). Sin embargo, no todas las personas que padecen esta enfermedad tienen el mismo nivel de discapacidad, incluso algunos han aprendido estrategias que les permiten reconocer a una persona vista previamente (Boucher y Lewis, 1992; Teunisse y De Gelder, 1994), lo que convierte en errónea la afirmación de que "las personas con TEA son incapaces de identificar a una persona". Tampoco parece útil basarse en la medida de inteligencia como índice de la capacidad para identificar a una persona, dado que algunas investigaciones muestran una escasa o nula correlación (Zhu, Song, Hu, Zhen, Dong, Kanwisher y Liu, 2009).

En cualquier caso, desde un punto de vista forense, lo relevante sería si esa víctima o testigo en concreto es capaz de identificar sin error a un agresor desconocido. La Psicología del Testimonio, como aplicación de los estudios sobre los procesos cognitivos a la obtención y valoración de la prueba testifical, ha analizado qué procesos son relevantes para la tarea testifical y cuál es el efecto que tienen sobre la exactitud de las identificaciones las características 
del testigo y del suceso, los factores de retención y recuperación, y otros específicos de los sistemas de investigación (para una revisión ver Manzanero, 2010).

El objetivo específico del presente trabajo consistió en analizar la capacidad de personas con discapacidad intelectual inespecífica ligera y moderada para identificar a personas desconocidas vistas durante un breve periodo de tiempo.

\section{Método}

Para evaluar la capacidad de las personas con DI para identificar a personas desconocidas vistas durante un breve periodo de tiempo se diseñó un experimento de un factor, con dos condiciones, personas con DI y control. Participaron en el estudio un total de 158 sujetos, 78 con discapacidad intelectual inespecífica ligera y moderada (CI medio de 60.91, $D T=10.77$ ), que conforman el grupo experimental y 80 sujetos sin discapacidad intelectual, que conforman el grupo control. En la tabla 1 se describe la muestra. El grupo experimental está compuesto por personas con discapacidad intelectual que trabajan en los talleres ocupacionales y centros especiales de empleo de la Fundación Carmen Pardo-Valcarce, de Madrid. El grupo control lo forman profesionales que trabajan en la misma institución (jefes de taller, profesores, psicólogos, cocineras, servicio de limpieza, etc).

Tabla 1. Muestra de sujetos participantes en el experimento

\begin{tabular}{cc}
\hline Control & DI \\
\hline Hombres: 42 & Hombres: 49 \\
Edad: 38.26 & Edad: 33.29 \\
$D T: 11.03$ & $D T: 7.11$ \\
& \\
Mujeres: 38 & Mujeres: 29 \\
Edad: 37.26 & Edad: 30.31 \\
$D T: 9.38$ & $D T: 6.19$
\end{tabular}

Las medidas utilizadas para ver el efecto de la variable independiente fueron la exactitud de las identificaciones (aciertos, falsas alarmas, omisiones y rechazos correctos), el índice de discriminabilidad d' y el criterio de respuesta $\beta$ en el marco de la Teoría de Detección de Señales (Tanner y Swets, 1954).

\section{Procedimiento}

Se utilizó un procedimiento adaptado del utilizado por Lyle y Johnson (2004). Individualmente para cada uno de los sujetos, se presentó una fotografía de un hombre o una mujer durante seis segundos, para después de una tarea de búsqueda visual con letras, de un minuto de duración, participar en dos ruedas de identificación (la primera de objetivo ausente, es decir, no aparece la persona a identificar entre las fotografías presentadas y la segunda de objetivo presente, en la que sí aparece la persona a identificar entre las demás fotografías). Las ruedas de reconocimiento estaban compuestas por seis fotografías cada una, presentadas de forma simultánea. Los sujetos debían responder para cada ensayo señalando si alguna de las fotografías correspondía con la presentada inicialmente o si no era ninguna. Cada sujeto participó en cuatro ensayos, dos con fotografías de hombre y dos con fotografías de mujer.

La fotografía objetivo presentada representaba a una persona joven (en torno a los 20 años de edad) de frente y vestida de negro para que la vestimenta no sirviera de indicio. Las fotografías distractoras eran de personas de características similares a las correspondientes fotografías objetivo, todas ellas morenas y blancas, sin rasgos distintivos especiales y también vestidas de negro. Los distractores fueron elegidos por su parecido físico con la fotografía objetivo de entre 452 fotografías de las fichas de alumnos de la Licenciatura en Psicología de la Universidad Autónoma de Madrid del curso 2002/2003.

Como tarea distractora se eligió una prueba de búsqueda visual con letras siguiendo el paradigma propuesto inicialmente por Neisser (1963). Se trata de una tarea habitualmente utilizada para evaluar la capacidad atencional de los sujetos y que por lo tanto aquí además nos permitirá descartar diferencias en esta capacidad entre los dos grupos de sujetos. Por otro lado, debido a que en ambas pruebas se le pide a los sujetos que realicen la misma tarea: seleccionar un estímulo previamente presentado de entre un conjunto de estímulos equiparables, la realización de la tarea de búsqueda visual serviría de entrenamiento para la tarea posterior de identifica- 
Figura 1. Procedimiento seguido en el experimento 1 (las fotografías se han difuminado para preservar su identidad)

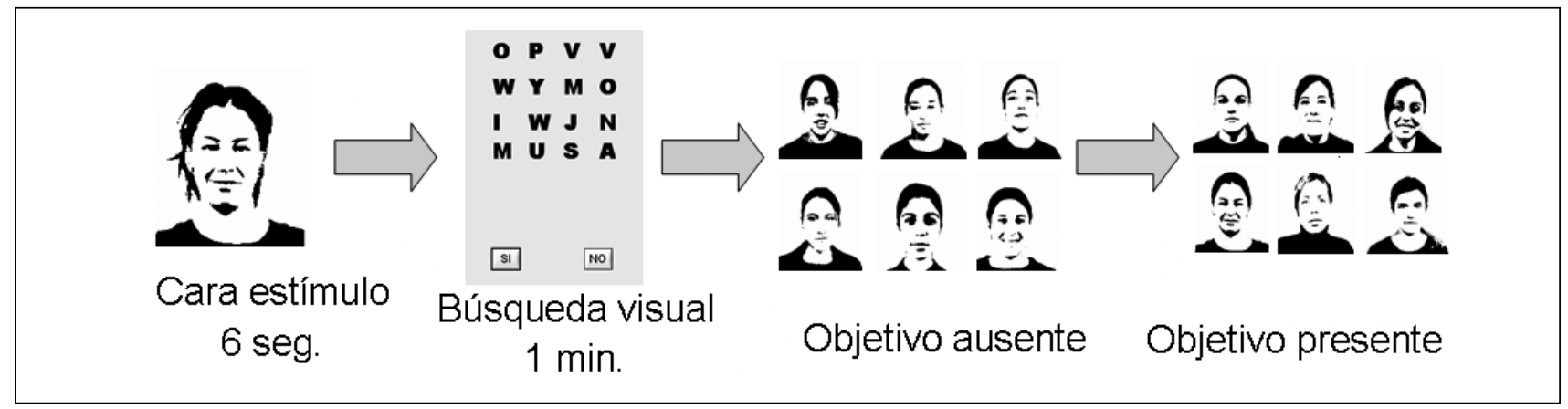

ción de personas, facilitando la comprensión de la misma. En cualquier caso, el rendimiento en ambas tareas no será comparable debido a que los estímulos presentados difieren en significado y familiaridad.

\section{Resultados}

\section{Tarea de búsqueda visual}

El porcentaje de aciertos en la tarea de búsqueda visual para los sujetos con DI fue del $89.39 \%$ mientras que el grupo control acertó en el $94.98 \%$ de los ensayos; $\mathrm{F}(1,156)=60.459, \mathrm{p}<0.001, \eta^{2}=0.281$, $1-\beta=1$. Sin embargo, no encontramos diferencias significativas en el porcentaje de falsas alarmas, aunque los sujetos con DI cometieron falsas alarmas en un $4.63 \%$ de los ensayos, por sólo un $0.96 \%$ de los sujetos control, $\mathrm{F}(1,156)=3.426, \mathrm{p}>0.05$.

El índice de discriminabilidad para esta tarea es menor para el grupo de personas con DI que para el grupo control (d' $=2.929$ vs. d' $=3.921$ respectivamente). No obstante, donde más diferencias encontramos es en el índice de criterio de respuesta, que nos indica la tendencia de los sujetos a señalar, y donde los sujetos con DI obtienen un valor de $\beta=$ 1.888 y los sujetos del grupo control de $\beta=4.477$ (puntuaciones menores que 1 indica un criterio liberal y mayores que 1 un criterio conservador).

\section{Tarea de identificación de caras}

En la tabla 2 se pueden observar las puntuaciones medias y proporciones para las medidas de aciertos, falsas alarmas, rechazos correctos y omisiones de los sujetos con DI (grupo experimental) y sin DI (grupo control) para las ruedas de objetivo ausente y objetivo presente.

En la rueda de sospechoso ausente, se encontró un $10.63 \%$ de falsas alarmas para el grupo control y un $27.33 \%$ para el grupo de sujetos con DI, $\chi^{2}(4)=$ $14.002, \mathrm{p}<0.000, \phi^{2}=0.298$ y un $89.38 \%$ de rechazos correctos para el grupo control y $72.67 \%$ para el grupo de sujetos con DI, $\chi^{2}(4)=14.494, p<0.01, \phi^{2}=0.303$.

En la rueda de objetivo presente, se encontró un $17.95 \%$ de falsas alarmas en el grupo de personas con DI y un $4.6 \%$ para el grupo control, $\chi^{2}(4)=$ 23.082 , $\mathrm{p}<0.000 ; \phi^{2}=0.382$; un $38.14 \%$ de omisiones en DI y un $15 \%$ en el grupo control, $\chi^{2}(4)=$ $29.424, \mathrm{p}<0.000, \phi^{2}=0.432$; y un $43.91 \%$ de aciertos en DI por un $80.94 \%$ en el grupo control; $\chi^{2}(4)$ $=59.291, \mathrm{p}<0.000, \phi^{2}=0.613$.

En el grupo control, de los 12 sujetos que en la primera rueda de objetivo ausente señalan a una persona, 7 sujetos vuelven a señalar erróneamente en la segunda rueda de objetivo presente (58.33\%). De los 38 sujetos del grupo de personas con DI que señalan a una persona en la rueda de objetivo ausente, 25 vuelven a señalar erróneamente a un cebo en la rueda de objetivo presente (65.78\%).

Los índices de discriminabilidad (d') y criterio de respuesta (ß) muestran que los sujetos con DI tienen problemas al discriminar las caras de las personas vistas previamente de las caras nuevas ( $\mathrm{d}^{\prime}=0.397$ vs. d' = 2.098), como puede apreciarse en las gráficas siguientes.

El criterio de respuesta muestra que tanto los sujetos con DI como los sujetos sin DI utilizan criterios conservadores, aunque el grupo control es más conservador $(\beta=1.134)$ que los sujetos con DI $(\beta=1.040)$. 
Tabla 2. Puntuaciones medias, desviaciones típicas y proporciones para cada tipo de respuesta en las ruedas de objetivo ausente y objetivo presente, discriminabilidad (d') y criterio de respuesta (ß)

\begin{tabular}{|c|c|c|c|}
\hline & $M$ & $D T$ & Prop \\
\hline \multicolumn{4}{|l|}{ Sujetos Control } \\
\hline \multicolumn{4}{|l|}{ Rueda de objetivo ausente } \\
\hline - Rechazos correctos & 3.58 & 0.74 & 89.38 \\
\hline • Falsas Alarmas & 0.43 & 0.74 & 10.63 \\
\hline \multicolumn{4}{|l|}{ Rueda de objetivo presente } \\
\hline - Aciertos & 3.24 & 0.85 & 80.94 \\
\hline • Omisión & 0.60 & 0.79 & 15 \\
\hline - Falsas Alarmas & 0.16 & 0.40 & 4.06 \\
\hline - d' & & 2.10 & \\
\hline - $\beta$ & & 1.13 & \\
\hline \multicolumn{4}{|l|}{ Sujetos con DI } \\
\hline \multicolumn{4}{|l|}{ Rueda de objetivo ausente } \\
\hline - Rechazos correctos & 2.90 & 1.40 & 72.67 \\
\hline • Falsas Alarmas & 1.09 & 1.41 & 27.33 \\
\hline \multicolumn{4}{|l|}{ Rueda de objetivo presente } \\
\hline - Aciertos & 1.76 & 1.12 & 43.91 \\
\hline • Omisión & 1.53 & 1.21 & 38.14 \\
\hline - Falsas Alarmas & 0.72 & 0.91 & 17.95 \\
\hline - d' & & 0.40 & \\
\hline - $B$ & & 1.04 & \\
\hline
\end{tabular}

\section{Relación entre Cociente de Inteligencia y rendimiento}

Aunque algunas investigaciones (Plesa-Skwerer, Faja, Schofield, Verbalis, Tager-Flusberg y Dykens, 2006; Zhu y cols., 2009) no encuentran relación entre el Cociente de Inteligencia (CI) y la capacidad para identificar caras, no es así en todas los estudios en los que se considera DI e identificación (López, Donnelly, Hadwin y Leekam, 2004).

Los datos del presente experimento (ver Tabla 3) nos indican que el CI correlaciona significativamente con las respuestas emitidas por las personas con DI en la rueda de objetivo ausente. Valores de CI más altos correlacionan positivamente con los rechazos correctos y negativamente con las falsas alarmas, de modo que a mayor CI menor es la probabilidad de señalar erróneamente a un inocente en la rueda de reconocimiento cuando no está presente el autor en la rueda.

Con respecto a la rueda de objetivo presente el CI únicamente correlaciona de forma significativa con los aciertos. A mayor CI mayor es la probabilidad de señalar correctamente a la persona vista previamente cuando está presente en la rueda. Sin embargo, en

Figura 2. Representación gráfica del solapamiento entre las distribuciones cebo (cara nueva) y estímulo objetivo (cara vista previamente) para los sujetos con DI (a) y control (b).

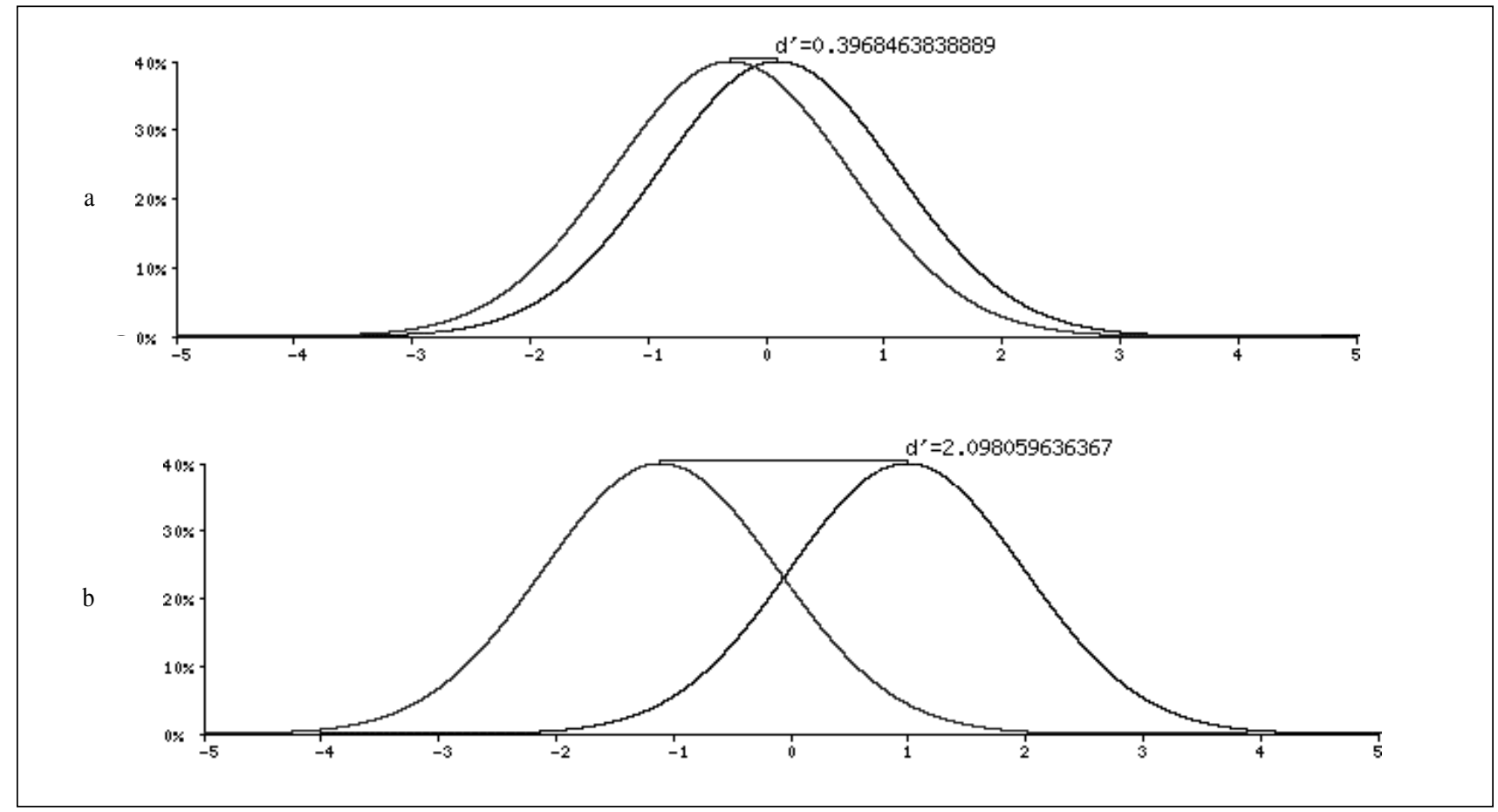


Tabla 3. Coeficientes de correlación de Pearson (bilateral) para CI y tipo de respuesta en el grupo de personas con DI

\begin{tabular}{|c|c|c|c|}
\hline & & $r$ & Sig. \\
\hline \multicolumn{4}{|l|}{ Rueda de objetivo ausente } \\
\hline & Rechazo correcto & 0.29 & $\mathrm{p}<0.05$ \\
\hline & Falsas alarmas & -0.32 & $\mathrm{p}<0.05$ \\
\hline \multicolumn{4}{|c|}{ Rueda de objetivo presente } \\
\hline & Aciertos & 0.35 & $\mathrm{p}<0.05$ \\
\hline & Falsas alarmas & -0.09 & $\mathrm{p}>0.05$ \\
\hline & Omisiones & -0.26 & $p>0.05$ \\
\hline
\end{tabular}

estas ruedas no hay correlación entre el CI y las falsas alarmas y omisiones.

\section{Discusión}

De los datos encontrados se desprende que los sujetos con DI tienden a presentar más problemas al tratar de identificar la fotografía de una persona desconocida vista durante un breve periodo de tiempo, en comparación con sujetos sin DI. Estos resultados confirman los encontrados en otras investigaciones previas (por ejemplo, Ericson y Isaacs, 2003). La pregunta es a qué se debe este déficit y si se puede minimizar su impacto, sobretodo en lo que se refiere a la presencia de falsas alarmas. Así, surgen varias posibles hipótesis: a) que se trate de un problema perceptivo, b) que se trate de un problema de comprensión de la tarea, c) que sea un problema atencional, y/o d) que se deba a un problema de aquiescencia.

Sobre la primera hipótesis, que sea un problema perceptivo, algunas investigaciones mostraron que las personas que presentan determinados síndromes (por ejemplo los trastornos de espectro autista) asociados a discapacidad intelectual tienen problemas en la capacidad para integrar la información, que afectaría al procesamiento holístico de caras (Joseph y Tanaka, 2003; López y cols., 2004). Siendo así, algunos sujetos con DI que presenten dichos síndromes podrían tener menos problemas al identificar rasgos que al identificar la cara de una persona como un todo. Aunque algunos otros síndromes (por ejemplo el síndrome de Williams) no parecen tener este déficit (Tager-Flusberg, Plesa-Skwerer, Faja y Joseph, 2003). Dado que la mayoría de las discapacidades intelectuales son de causa inespecífica, no podrían generalizarse los resultados.
Con respecto a la capacidad de comprensión de la tarea, los datos procedentes de la prueba de búsqueda visual, mostraron diferencias entre los dos grupos únicamente en los aciertos. Los altos porcentajes de aciertos en el grupo de personas con DI y la falta de diferencias en las falsas alarmas podrían indicar que el rendimiento encontrado en la tarea de identificación de caras no se debe a un déficit en la comprensión de la tarea. Por otro lado, el índice de discriminabilidad para la tarea de la búsqueda visual de las letras, fue mayor en los dos grupos, en comparación a los obtenidos para la tarea de identificación de caras, siendo la capacidad para discriminar letras mayor en el grupo control que en el grupo experimental. No obstante, donde más diferencias se encontraron fue en el índice de criterio de respuesta, observándose que los sujetos con DI utilizaban criterios de respuesta menos conservadores que los sujetos del grupo control, lo que podría explicar, al menos en parte, la diferencia en rendimiento, dado que ni por los datos aquí recogidos ni por investigaciones previas (Zhu y cols., 2009) quepa suponer que el rendimiento se deba a un déficit atencional. Aunque esta capacidad es una de las que se ve frecuentemente alterada en los sujetos con DI (Brown, Johnson, Peterson, Gilmore, Longui, KarmiloffSmith, 2003; Tomporowski, 1997).

Así, las diferencias en rendimiento probablemente puedan deberse a un problema de aquiescencia, lo que podría explicar que sólo se encuentre correlación entre el CI y las falsas alarmas en la rueda de objetivo ausente. En este sentido, es numerosa la literatura que nos indica que las personas con discapacidad intelectual son especialmente sensibles a la aquiescencia (Rapley y Antaki, 1996).

\section{Conclusiones}

La principal cuestión surge con respecto a las maneras de proceder con personas con discapacidad intelectual en las ruedas de reconocimiento, en aquellos contextos especialmente difíciles, donde el contacto con el agresor ha sido muy breve.

La primera duda era aquella planteada sobre la relación entre discapacidad intelectual y dificultad en el reconocimiento de caras. El presente estudio 
muestra que, efectivamente, en situaciones en las que el tiempo de exposición de la cara objetivo es breve, las personas con DI tienen más dificultades en su reconocimiento. El dato más preocupante, de cara a la aplicación práctica, es el riesgo de obtener un mayor número de falsas alarmas que las personas sin DI si no se adecua el contexto forense a la realidad de la personas con DI: una realidad muchas veces marcada por un déficit en los procesos atencionales y de memoria, y por una tendencia a mostrar respuestas aquiescentes por la necesidad de deseabilidad social, que se incrementa cuanta más inseguridad encuentre en la tarea.

Si se pudiera crear un contexto en el que la persona con DI se encontrara más segura con la tarea, más motivada para realizarla adecuadamente, y con una mayor conciencia de la importancia de evitar los falsos positivos, probablemente podríamos conseguir mejores resultados. Siguientes investigaciones se encaminarán en este sentido.

\section{Agradecimientos}

La presente investigación ha sido posible gracias a la participación de los profesionales de la Fundación Carmen Pardo-Valcarce así como de los usuarios que cada día trabajan en ella. El trabajo se enmarca en el proyecto "Entrevista, intervención y criterios de credibilidad en abusos de carácter sexual en personas con discapacidad intelectual" financiado por la Fundación MAPFRE.

\section{Referencias}

Annaz, D., Karmiloff-Smith, A., Johnson, M. H. y Thomas, M. S. C. (2009). A cross-syndrome study of the development of holistic face recognition in children with autism, Down syndrome and Williams syndrome. Journal of Experimental Child Psychology, 102, 456-486

Behrmann, M., Avidan, G., Leonard, G. L., Kimchi, R., Luna, B., Humphreys, K. y Minshew, N. (2006). Configural processing in autism and its relationship to face processing. Neuropsychologia, $44,110-129$.
Boucher, J. y Lewis, V. (1992). Unfamiliar face recognition in relatively able autistic children. Journal of Child Psychology and Psychiatry, 33, 843-859.

Brown, J., Johnson, H., Peterson, S., Gilmore, R., Longui, E. y Karmiloff-Smith, A. (2003). Spatial representation and attention in toodlers with Williams Syndrome and Down Syndrome. Neuropsychologia, 41, 1037-1046

Davies, S., Bishop, D., Manstead, A. y Tantam, D. (1994). Face perception in children with autism and Asperger's syndrome. Journal of Child Psychology and Psychiatry, 35, 1033-1057.

Ericson, K. y Isaacs, B. (2003). Eyewitness identification accuracy: A comparison of adults with and those without intellectual disabilities. Mental Retardation, 41, 161-173.

Gepner, B., De Gelder, B. y Schonen, S. (1996). Face processing in autistics: Evidence for a generalised deficit? Child Neuropsychology, 2, 123-139.

Goldman, R. L. (1994). Children and youth with intellectual disabilities: Targets for sexual abuse. International Journal of Disability, Development and Education, 41, 89-102.

Joseph, R. y Tanaka, J. (2003). Holistic and partbased face recognition in children with autism. Journal of Child Psychology and Psychiatry, 44, 529-542.

López, B., Donnelly, N., Hadwin, J. A. y Leekam, S. R. (2004). Face processing in high-functioning adolescents with autism: Evidence for weak central coherence. Visual Cognition, 11, 676-688.

Lawrence, K., Kuntsi, J., Coleman, M., Campbell, R. y Skuse, D. (2003). Face and emotion recognition deficits in Turner syndrome: A possible role for X-linked genes in amygdala development. Neuropsychology, 17, 39-49.

Lyle, K. B. y Johnson, M. K. (2004). Effects of verbalization on lineup face recognition in an interpolated inspection paradigm. Applied Cognitive Psychology, 18, 393-403.

Manzanero, A. L. (2006). Las experiencias de recuperación como medida de memoria. Boletín de Psicología, 87, 89-105.

Manzanero, A. L. (2010). Memoria de Testigos. Obtención y valoración de la prueba testifical. Madrid: Pirámide. 
Neisser, U. (1963). Decision time without reaction time: Experiments in visual scanning. American Journal of Psychology, 76, 376-385

Plesa-Skwerer, D., Faja, S., Schofield, C., Verbalis, A., Tager-Flusberg, H. y Dykens, E. M. (2006). Perceiving facial and vocal expressions of emotion in individuals with Williams Syndrome. American Journal on Mental Retardation, 111, 15-26.

Rapley, M. y Antaki, C (1996). A conversation analysis of the 'acquiescence' of people with learning disabilities. Journal of Community and Applied Psychology, 6, 207-227.

Tager-Flusberg, H., Plesa-Skwerer, D., Faja, S. y Joseph, R. M. (2003). People with Williams syndrome process faces holistically. Cognition, 89, 11-24.
Tanner, W. P. y Swets, J. A. (1954). A decision-making theory of visual detection. Psychological Review, 61, 401-409.

Teunisse, J. P. y De Gelder, B. (1994). Do autistics have a generalized face processing deficit? International Journal of Neuroscience, 77, 110.

Tomporowsky, P. (1997). Attention in mentally retarded persons. En W. Mac Lean (Ed.), Handbook of mental deficiency, psychological theory and research. New Jersey: LEA.

Zhu, Q., Song, Y., Hu, S., Li, X., Tian, M., Zhen, Z., Dong, Q., Kanwisher, N. y Liu, J. (2009). Heritability of the specific cognitive ability of face perception. Current Biology, 20, 137-142.

Manuscrito recibido:10/01/2011

Revisión recibida: 21/02/2011

Aceptado: 24/02/2011 